\title{
EL CUIDADO TRANSICIONAL DE ENFERMERÍA AUMENTA LA COMPE- TENCIA EN EL ROL DEL CUIDADOR DEL NIÑO CON CÁNCER
}

\author{
TRANSITIONAL CARE OF NURSING IMPROVES COMPETENCY IN THE ROLE \\ CAREGIVER OF CHILD WITH CANCER
}

\begin{abstract}
Sonia Carreño Moreno
Facultad de Enfermería-Grupo de Investigación Cuidado de Enfermería al paciente crónico, Bogotá, Colombia.
\end{abstract}

Resumen

Introducción. El cuidador familiar del niño con cáncer pasa por una transición en su rol en la que requiere fortalecer su competencia para ejercerlo.

Objetivo. Determinar el efecto de una intervención de cuidado transicional de enfermería sobre la competencia de cuidado del cuidador familiar del niño con cáncer.

Materiales y métodos. Estudio experimental, el grupo experimental $(n=59)$ recibió la intervención de cuidado transicional de enfermería "cuidando a nuestros niños con cáncer" y el grupo control $(n=59)$ la atención convencional de la institución de salud. Se midió la competencia del cuidador familiar para el cuidado, se trató de un estudio doble ciego. El estudio contó con el aval de comités de ética institucional y llevó a cabo consentimiento informado. El análisis se realizó con las pruebas $T$ de student y diferencia de medias estanadarizada d de Cohen.

Resultados. La mayoría de los niños tuvieron diagnóstico de Leucemia $41 \%$, con edad media de 4 años. Los cuidadores familiares son mujeres $84 \%$, con edad media de 33 años. Se observó una diferencia entre la competencia para cuidar $(p<0,001)$ entre el grupo experimental y control en la postratamiento, con un tamaño del efecto $d=5,14$.

Conclusión. La intervención "Cuidando a nuestros niños con cáncer" tiene un efecto
Abstract

Introduction. The family caregiver of child with cancer goes through a transition in their role in strengthening competence required to exercise it.

Objective. To determine the effect of an intervention of transitional nursing care on the competency of the family caregiver of a child with cáncer.

Methodology. Experimental study, the experimental group $(n=59)$ underwent an intervention developed by the investigators "Taking care of our children with cancer", the control group $(n=59)$ received usual care. It was measured the competency of care of the caregiver, it was a double-blind study. The analysis was performed using the Student's t test, the effect size was estimated using average exchange standardized difference - d Cohen.

Results. Most of the children were diagnosed with leukemia $41 \%$ and the average age was 4 years. Family caregivers are women $84 \%$, mean age 33. A competency of care difference $(p<0.001)$ was observed in the posttest, with an effect size $d=5.14$

Conclusion. The intervention "Caring for our children with cancer" has strong effect increasing the competency of care of family caregiver in the transition process of their role. The intervention is applicable to practical and replicable in the investigation.

\section{Correspondencia:}

Sonia Carrero Moreno

Facultad de Enfermería

Grupo de Investigación Cuidado de Enfermería al paciente crónico. Universidad Nacional de Colombia.

Carrera 30 No.45 - 03, Edif. 228, Bogotá, Código Postal: 111321 - Colombia.

E-mail: spcarrenom@unal.edu.com 
fuerte en el aumento de la competencia para el cuidado del cuidador familiar en el proceso de transición del su rol. La intervención es aplicable a la práctica y replicable en la investigación.

Palabras Clave: Family caregivers, child care, oncology nursing, health transition.
Keywords: Family caregivers, child care, oncology nursing, health transition.

\section{INTRODUCCIÓN}

El cáncer infantil es una afección que impacta no solo al niño que lo padece sino también a sus allegados y en especial a su cuidador familiar. El cuidador familiar del niño con cáncer es por lo general su madre o padre, este dedica gran parte de su tiempo al cuidado ${ }^{(1)}$ y vive una transición en su rol, pues pasa de ser la madre o padre de un niño sano a ser el cuidador de un niño con cáncer ${ }^{(2)}$.

La transición del rol en el cuidador del niño con cáncer, se caracteriza por ser una experiencia en la que éste percibe que posee pocos conocimientos y preparación para ejercer su función ${ }^{(3-5)}$, situación que implica falta de satisfacción con los sentimientos y metas asociadas al desempeño del rol y falta de suficiencia para desarrollarlo ${ }^{(6)}$.

La insuficiencia del rol del cuidador obstaculiza la progresión hacia una transición adecuada ${ }^{(6)}$ y se relaciona con condiciones que pueden facilitarla o inhibirla. Como parte del proceso, existe una condición que es clave a la hora de intervenir al cuidador del niño con cáncer: la preparación y conocimiento(7). En este aspecto, los cuidadores necesitan orientación y una amplia gama de información que incluye la patología, el tratamiento, efectos secundarios, afrontamiento, cuidado en el hogar, redes de apoyo social y toma de decisiones ${ }^{(8)}$.

Los cuidadores familiares de niños con cáncer reconocen en enfermería una profesión idónea que los puede preparar en la transición ${ }^{(9)}$, lo que permite hipotetizar que una intervención de cuidado transicional de enfermería puede incidir sobre la condición de preparación y conocimiento en el cuidador en términos de su competencia para cuidar y por tanto lograr una transición adecuada para el ejercicio del rol.

El objetivo de este estudio fue determinar el efecto de una intervención de cuidado transicional de enfermería sobre la competencia de cuidado del cuidador familiar del niño con cáncer que es atendido en el Instituto Nacional de Cancerología E.S.E. Colombia.

\section{MATERIALES Y MÉTODOS}

Estudio experimental con grupo experimental y control, con mediciones pre prueba y pos prueba.

Se realizó un muestreo probabilístico aleatorio. Se calculó un tamaño de muestra de 59 participantes en el grupo experimental y 59 en el grupo control, con una probabilidad de error tipo 1 de 0,005 y tipo 2 de 0,02.

Fueron criterios de inclusión: ser cuidador familiar de un niño con cáncer, ser mayor de 18 años, estatus mental intacto valorado por SPMSQ, saber leer y escribir, tener como parentesco madre o padre.

Fueron excluidos cuidadores de niños que se encontraron en momentos críticos de la enfermedad (Exacerbación de síntomas, exacerbación de efectos secundarios del tratamiento, previo a cirugía o en fase paliativa). 
Al grupo experimental se ofreció la intervención de cuidado transicional de enfermería "cuidando a nuestros niños con cáncer" ver tabla 1.

La intervención fue diseñada con base en la propuesta de Meleis ${ }^{(2)}$, en donde se considera la transición en el rol como un evento en el que intervienen diferen- tes condiciones que pueden facilitarla e inhibirla. La condición de interés en la intervención fue la preparación y conocimiento del cuidador del niño con cáncer, expresada en términos de su competencia para cuidar. La intervención se desarrolló considerando la forma como se aprenden los roles, incluyendo así, actividades de

\section{Tabla 1. Protocolo de intervención}

\begin{tabular}{|c|c|c|}
\hline \multicolumn{3}{|c|}{ Nivel individual. Se realiza en el hospital, en la habitación de hospitalización. } \\
\hline Encuentro & Actividades & Material \\
\hline $\begin{array}{l}\text { Encuentro } 1 . \\
\text { Introducción } \\
\text { al programa } \\
\text { (Instrucción } \\
\text { intencional) }\end{array}$ & $\begin{array}{l}\text { Presentación del participante y espacio de } \\
\text { interacción con la enfermera para propiciar } \\
\text { el inicio de la relación terapéutica. } \\
\text { Socialización del objetivo y contenido del } \\
\text { programa. } \\
\text { Entrega del material escrito: Cartilla } \\
\text { "cuidando a nuestros niños con cáncer", } \\
\text { la cual contiene información de cada } \\
\text { encuentro, escrita en forma sencilla y breve. } \\
\text { Instrucción de lectura del Capítulo 1, 3, 4, } \\
5,7,8,9 \text { de la cartilla "Cuidando a nuestros } \\
\text { niños con cáncer en el hogar" }\end{array}$ & $\begin{array}{l}\text { Cartilla cuidando a } \\
\text { nuestros niños con cáncer } \\
\text { en el hogar }\end{array}$ \\
\hline $\begin{array}{l}\text { Encuentro } 2 . \\
\text { Conocimiento } \\
\text { y habilidades } \\
\text { instrumentales a } \\
\text { desarrollar en el } \\
\text { cuidador familiar } \\
\text { del niño con } \\
\text { cáncer (Instrucción } \\
\text { intencional, ensayo } \\
\text { del rol) }\end{array}$ & $\begin{array}{l}\text { Contenido: el cáncer infantil, tratamiento, } \\
\text { efectos secundarios del tratamiento contra } \\
\text { el cáncer, necesidades del niño con cáncer, } \\
\text { actividad física, manejo de síntomas, } \\
\text { cuidados de la boca, medidas de higiene, } \\
\text { nutrición, signos de alarma en el niño con } \\
\text { cáncer. } \\
\text { Metodología: Iniciar solicitando expresar } \\
\text { las dudas que tiene respecto a la lectura } \\
\text { de la cartilla y resolverlas. Continuar con } \\
\text { el reforzamiento y fijación cognitiva de } \\
\text { aspectos clave de esta temática, formulando } \\
\text { al cuidador familiar las siguientes preguntas: } \\
\text { ¿Qué es el cáncer? } \\
\text { ¿Qué tipo de cáncer tiene el niño al que } \\
\text { cuida? } \\
\text { ¿En qué consiste el tratamiento que recibe y } \\
\text { para qué sirve? } \\
\text { ¿Qué cuidados requiere el niño y cómo los } \\
\text { Ilevaría a cabo? } \\
\text { Identificar puntos que necesiten refuerzo } \\
\text { a partir de las respuestas del cuidador y } \\
\text { ampliarlos. }\end{array}$ & $\begin{array}{l}\text { Capítulo1: Generalidades } \\
\text { del cáncer infantil } \\
\text { Capítulo 3. Actividad física } \\
\text { en el niño con cáncer. } \\
\text { Capítulo 4. Cuidados de la } \\
\text { boca en el niño con cáncer. } \\
\text { Capítulo } 5 . \quad \text { Medidas } \\
\text { básicas de higiene en el } \\
\text { niño con cáncer. } \\
\text { Capítulo 7. Nutrición en el } \\
\text { niño con cáncer. } \\
\text { Capítulo 8. Manejo de los } \\
\text { efectos secundarios del } \\
\text { tratamiento del cáncer. } \\
\text { Capítulo 9. Signos de alarma } \\
\text { en el niño con cáncer. }\end{array}$ \\
\hline
\end{tabular}




\begin{tabular}{|c|c|c|}
\hline $\begin{array}{l}\text { Encuentro } 3 . \\
\text { Habilidades } \\
\text { psicosociales a } \\
\text { desarrollar en el } \\
\text { cuidador el niño con } \\
\text { cáncer } \\
\text { (Clarificación ensayo } \\
\text { del rol) }\end{array}$ & $\begin{array}{l}\text { Contenido: afrontamiento, resolución de } \\
\text { problemas, comunicación, autocuidado, } \\
\text { relevo, redes de soporte social. } \\
\text { Metodología: Iniciar solicitando expresar } \\
\text { las dudas que tiene respecto a la lectura } \\
\text { de la cartilla y resolverlas. Continuar con } \\
\text { el reforzamiento y fijación cognitiva de } \\
\text { aspectos clave de esta temática. } \\
\text { Luego, contarle al cuidador los siguientes } \\
\text { casos hipotéticos: } \\
\text { Es momento de iniciar una nueva } \\
\text { quimioterapia a su hijo, usted debe firmar el } \\
\text { consentimiento para que se la inicien. } \\
\text { Luego de la quimioterapia empiezan a } \\
\text { aparecer síntomas en su hijo como náusea, } \\
\text { caída del cabello y esto le produce tristeza. } \\
\text { Le van a dar salida a su hijo. } \\
\text { De acuerdo con las situaciones presentadas, } \\
\text { ¿Qué habilidades requiere para afrontarla y } \\
\text { como las usaría? } \\
\text { Por último preguntarle: } \\
\text { ¿Qué acciones para su propio cuidado } \\
\text { planea y a qué plazo piensa que las puede } \\
\text { cumplir? } \\
\text { Terminar solicitándole al cuidador que } \\
\text { "Diligenciemos juntos su agenda de apoyo". }\end{array}$ & $\begin{array}{l}\text { Entregar "Mi agenda de } \\
\text { apoyo" que contiene } \\
\text { información sobre las } \\
\text { personas o instituciones } \\
\text { que pueden brindar apoyo } \\
\text { social al cuidador. La } \\
\text { agenda tendrá información } \\
\text { sobre: el nombre de la } \\
\text { persona o institución, } \\
\text { ubicación, qué apoyo me } \\
\text { puede brindar, números } \\
\text { telefónicos. } \\
\text { Capítulo2. Consideraciones } \\
\text { psicológicas del cáncer. }\end{array}$ \\
\hline \multicolumn{3}{|c|}{ Nivel grupal. Se realiza en un salón del hospital. } \\
\hline Encuentro & Actividades & Material \\
\hline $\begin{array}{l}\text { Encuentro 4. Sesión } \\
\text { de reforzamiento e } \\
\text { interacción con un } \\
\text { grupo de cuidadores } \\
\text { (Clarificación, } \\
\text { ensayo, } \\
\text { modelamiento del } \\
\text { rol e interacción con } \\
\text { otros) }\end{array}$ & $\begin{array}{l}\text { Contenido: Esta sesión pretende reforzar a } \\
\text { partir de material audiovisual y aclaración } \\
\text { de dudas, los contenidos vistos en las } \\
\text { sesiones precedentes. } \\
\text { Metodología: Presentar el video "Cuidando } \\
\text { a nuestros niños con cáncer en el hogar". } \\
\text { Preguntar acerca de dudas que se tengan y } \\
\text { resolverlas. } \\
\text { Entregar una copia del video, para que los } \\
\text { cuidadores dispongan de ella en el momento } \\
\text { que lo necesiten. }\end{array}$ & $\begin{array}{l}\text { Video "Cuidando a } \\
\text { nuestros niños con cáncer } \\
\text { en el hogar" }\end{array}$ \\
\hline
\end{tabular}

Fuente: Construcción propia 2015.

instrucción intencional, clarificación, modelamiento y ensayo del rol, además de la interacción con otros cuidadores como actores significativos en el proceso(2).

Al grupo control se le ofreció la atención convencional de la institución que consistió en educación a demanda por enfermería que incluye la información sobre la enfermedad, el tratamiento, los efectos secundarios, los signos de alarma, los cuidados generales y los trámites administrativos. 
La figura 1, presenta el diagrama de flujo del reclutamiento, la inclusión y el seguimiento de los participantes.

El estudio contó con el aval de comités de ética de la Facultad de Enfermería de la Universidad Nacional de Colombia y el del Instituto Nacional de Cancerología; se llevó a cabo consentimiento informado.

La competencia del cuidador familiar para el cuidado fue medida en la pre y pos prueba, se trató de un estudio doble ciego. La medición pos prueba se realizó un mes después de terminada la intervención, con el instrumento denominado "Competencia del Cuidado en el hogar del cuidador familiar de una persona con enfermedad crónica - CUIDAR"(10), que consta de 60 ítems que se miden con una escala tipo Likert con puntuaciones que van de 1 a 4, contiene 6 sub-escalas así:

Figura 1. Diagrama reclutamiento, seguimiento y análisis de participantes del estudio

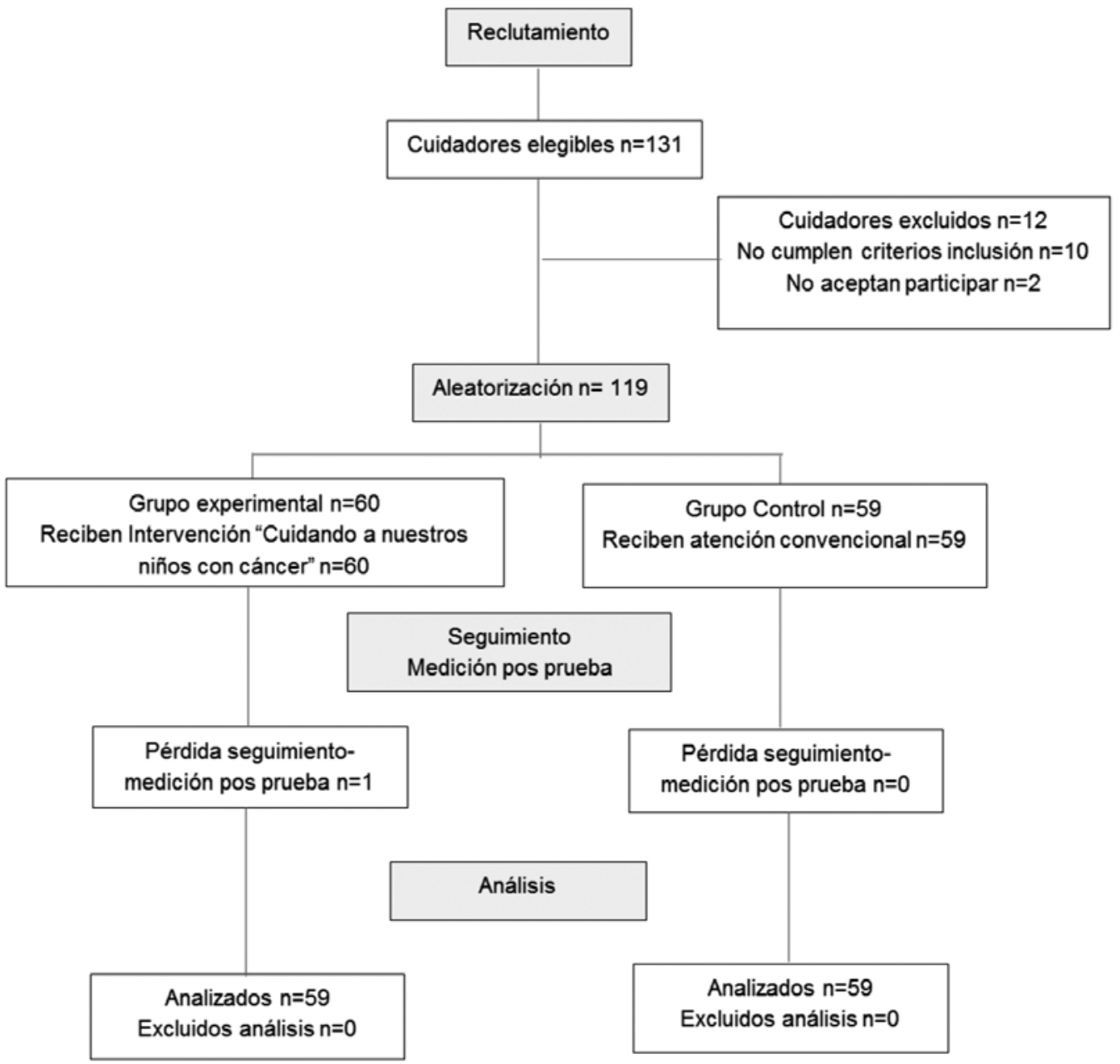

Fuente: Datos de estudio 2015. 
conocimiento, condiciones personales, instrumental, bienestar, anticipación, y relación social e interacción. El instrumento cuenta con validez facial, de contenido y de constructo. Cuenta además con confiabilidad por consistencia interna alfa de Cronbach de 0,97 para la totalidad del instrumento, 0,92 en la dimensión conocimiento, 0.89 condiciones personales, 0.89 Instrumental, 0,87 bienestar, 0,88 anticipación y 0,89 relación social e interacción ${ }^{(10)}$. Se realizó análisis de la consistencia interna con la muestra de este estudio (118 participantes), resultando un alfa de Cronbach global de 0,972.

Para el análisis de las variables de caracterización de la díada cuidador -niño con cáncer se usó estadística descriptiva. El análisis de la competencia para el cuidado se realizó con la prueba $t$ de student para muestras independientes. La estimación del tamaño del efecto se calculó con el método de diferencia de medias estandarizada, también conocida como $d$ de
Cohen, la cual informa las desviaciones típicas de diferencia entre los resultados del grupo experimental y control.

\section{RESULTADOS}

Características de los niños con cáncer

En la tabla 2 se observan las características de los niños con cáncer, receptores de cuidador de los cuidadores familiares incluidos en el estudio.

Características de los cuidadores

Las características de los cuidadores incluidos en el estudio son presentadas en la tabla 3 .

Comparación de la competencia para el cuidado

La competencia para el cuidado en los dos grupos antes de la intervención fue ho-

Tabla 2. Características de los niños con cáncer

\begin{tabular}{|c|c|c|c|}
\hline \multicolumn{2}{|c|}{ Características } & Experimental $\mathbf{n = 5 9}$ & Control $\mathbf{n = 5 9}$ \\
\cline { 2 - 4 } & Leucemias & Porcentaje & Porcentaje \\
\cline { 2 - 4 } & Linfomas & 37 & 45,8 \\
\cline { 2 - 4 } Diagnósticos médicos & Tumor sólido & 3 & 5,1 \\
\cline { 2 - 4 } & Tumor cerebral & 44 & 45,8 \\
\cline { 2 - 4 } & Femenino & 15 & 3,4 \\
\cline { 2 - 4 } Género & Masculino & 41 & 52,5 \\
\hline \multirow{3}{*}{$\begin{array}{c}\text { Grado máximo de } \\
\text { escolaridad }\end{array}$} & Ninguno & 39 & 47,5 \\
\cline { 2 - 4 } & Primaria & 31 & 33,9 \\
\cline { 2 - 4 } & Secundaria & 37 & 47,5 \\
\hline \multirow{2}{*}{ Ocupación } & Estudiante & 56 & 18,6 \\
\cline { 2 - 4 } & Hogar & 44 & 40,7 \\
\hline \multirow{2}{*}{ Edad } & Media & 9,07 & 59,3 \\
\cline { 2 - 4 } & Desviación estándar & 4,9 & 8,05 \\
\hline
\end{tabular}

Fuente: Datos de estudio 2015. 
Tabla 3. Características de los cuidadores de niños con cáncer

\begin{tabular}{|c|c|c|c|}
\hline \multicolumn{2}{|c|}{$\begin{array}{l}\text { Características } \\
\text { Porcentaje }\end{array}$} & \multirow{2}{*}{$\begin{array}{c}\begin{array}{c}\text { Grupo } \\
\text { experimental } \\
n=59\end{array} \\
\text { Porcentaje } \\
\end{array}$} & \multirow[t]{2}{*}{ Grupo control $n=59$} \\
\hline \multirow{3}{*}{ Género } & & & \\
\hline & Femenino & 83,1 & 84,7 \\
\hline & Masculino & 16,9 & 15,3 \\
\hline \multirow{6}{*}{$\begin{array}{l}\text { Grado máximo de } \\
\text { escolaridad }\end{array}$} & Ninguno & 1,7 & 3,4 \\
\hline & Primaria & 44,1 & 28,8 \\
\hline & Pregrado & 10,2 & 3,4 \\
\hline & Posgrado & 0,0 & 1,7 \\
\hline & Secundaria & 42,4 & 57,6 \\
\hline & Técnico & 1,7 & 5,1 \\
\hline \multirow{5}{*}{ Estado Civil } & Casado(a) & 32,2 & 23,7 \\
\hline & Separado (a) & 5,1 & 5,1 \\
\hline & Soltero(a) & 23,7 & 25,4 \\
\hline & Unión libre & 33,9 & 44,1 \\
\hline & Viudo(a) & 5,1 & 1,7 \\
\hline \multirow{4}{*}{ Ocupación } & Empleado(a) & 15,3 & 15,3 \\
\hline & Estudiante & 5,1 & 6,8 \\
\hline & Hogar & 59,3 & 67,8 \\
\hline & $\begin{array}{c}\text { Trabajo } \\
\text { independiente }\end{array}$ & 20,3 & 10,2 \\
\hline \multirow{2}{*}{ Edad } & Media & 33,5 & 33,1 \\
\hline & DT & 7,3 & 6,5 \\
\hline \multirow{2}{*}{$\begin{array}{l}\text { Tiempo en la relación de } \\
\text { cuidado en meses }\end{array}$} & Media & 9,92 & 5,56 \\
\hline & DT & 11,08 & 5,55 \\
\hline \multirow{2}{*}{$\begin{array}{c}\text { Número de horas diarias de } \\
\text { cuidado }\end{array}$} & Media & 22,20 & 23,59 \\
\hline & DT & 4,58 & 1,92 \\
\hline
\end{tabular}

Fuente: Datos de estudio 2015.

moscedástica valorada a través de la prueba de Levene con un valor $p$ de 0,115, en consecuencia la prueba $t$ de Student bajo esta condición produjo un valor de la estadística de la prueba de 0,775 y un valor $\mathrm{p}$ asociado de 0,44 , lo cual indica que los dos grupos eran similares antes de la intervención.

Por el contrario, la competencia para el cuidado en los grupos después de la intervención fue heteroscedástica valorada a través de la prueba de Levene con un va- lor $p<0,001$, en consecuencia la prueba $t$ de Student se basó en aproximación de Welch, con una modificación de los grados de libertad de 86,781. Esta prueba advierte que la competencia media entre los dos grupos al término de la intervención difiere, con una significancia estadística $p<0,001$.

El resumen de la prueba, la media y desviación típica de los grupos experimental y control en la pre y pos prueba de estudio son presentados en la tabla 4, frente al total del instrumento y sus subescalas. 
Tabla 4. Estadísticos comparación intergrupos competencia para el cuidado del cuidador - CUIDAR pre y pos prueba

\begin{tabular}{|c|c|c|c|c|c|c|}
\hline \multirow{2}{*}{ Grupo /Estadístico } & \multicolumn{3}{|c|}{ PREPRUEBA } & \multicolumn{3}{|c|}{ POSPRUEBA } \\
\hline & Media & Desv. Típica & $\mathrm{P}$ valor & Media & Desv. Típica & $\mathrm{P}$ valor \\
\hline \multicolumn{7}{|c|}{ TOTAL CUIDAR } \\
\hline Experimental & 153,93 & 9,68 & \multirow{2}{*}{0,440} & 216,75 & 6,20 & \multirow{2}{*}{$<0,001$} \\
\hline Control & 152,39 & 11,81 & & 159,29 & 12,03 & \\
\hline \multicolumn{7}{|c|}{ SUBESCALA CONOCIMIENTO } \\
\hline Experimental & 24,02 & 3,46 & \multirow{2}{*}{0.746} & 36,33 & 1,84 & \multirow{2}{*}{$<0.001$} \\
\hline Control & 23,81 & 3,59 & & 25,01 & 3,01 & \\
\hline \multicolumn{7}{|c|}{ SUBESCALA CONDICIONES PERSONALES } \\
\hline Experimental & 30,05 & 2,98 & \multirow{2}{*}{0.638} & 42,69 & 2,01 & \multirow{2}{*}{$<0.001$} \\
\hline Control & 30,10 & 3,38 & & 30,84 & 3,96 & \\
\hline \multicolumn{7}{|c|}{ SUBESCALA INSTRUMENTAL } \\
\hline Experimental & 20,18 & 2,96 & \multirow{2}{*}{$<0.001$} & 28,38 & 1,82 & \multirow{2}{*}{$<0.001$} \\
\hline Control & 21,59 & 1,64 & & 22,66 & 2,51 & \\
\hline \multicolumn{7}{|c|}{ SUBESCALA BIENESTAR } \\
\hline Experimental & 31,38 & 3,72 & \multirow{2}{*}{$<0.001$} & 43,57 & 1,85 & \multirow{2}{*}{$<0.001$} \\
\hline Control & 33,40 & 2,77 & & 35,94 & 3,42 & \\
\hline \multicolumn{7}{|c|}{ SUBESCALA ANTICIPACIÓN } \\
\hline Experimental & 16,57 & 2,13 & \multirow{2}{*}{$<0.001$} & 22,11 & 1,36 & \multirow{2}{*}{$<0.001$} \\
\hline Control & 14,61 & 2,59 & & 14,81 & 2,79 & \\
\hline \multicolumn{7}{|c|}{ SUBESCALA RELACIÓN SOCIAL E INTERACCIÓN } \\
\hline Experimental & 31,71 & 2,49 & \multirow{2}{*}{0.007} & 43,62 & 2,11 & \multirow{2}{*}{$<0.001$} \\
\hline Control & 28,86 & 5,14 & & 30 & 4,98 & \\
\hline
\end{tabular}

Fuente: Datos de estudio 2015.

ESTIMACIÓN DEL TAMAÑO DEL EFECTO

Todas las dimensiones y la competencia total, presentaron valores de diferencia de medias estandarizada o $d$ de
Cohen mayores a 2, lo que muestra un cambio pos prueba de gran magnitud en la competencia para el cuidado y sus dimensiones.

El resumen del cálculo de tamaño del efecto se presenta en la tabla 5.

\section{Tabla 5. Cálculo del tamaño de efecto d de Cohen, total CUIDAR y dimensiones}

\begin{tabular}{|c|c|}
\hline Variable & Diferencia de medias estandarizada (d Cohen) \\
\hline Conocimiento & 3,13 \\
\hline Condiciones personales & 3,70 \\
\hline Instrumental y procedimental & 2,96 \\
\hline Bienestar & 2,92 \\
\hline Anticipación & 2,23 \\
\hline Relación social e interacción & 2,65 \\
\hline Total Competencia & 5,14 \\
\hline
\end{tabular}




\section{DISCUSIÓN}

El tiempo medio en la relación de cuidado de las díadas cuidador-niño con cáncer estuvo cerca de los 7 meses y medio, situación relacionada con las características propias de la muestra, pues son cuidadores familiares de niños que están en los primeros meses de tratamiento oncológico. Se observó una alta dedicación en horas diarias al cuidado las cuales oscilaron entre 22 y 24 horas. La mayoría de niños tienen diagnósticos oncológicos que hacen parte de las leucemias y tumores sólidos, situación que es coherente con el tipo de neoplasias que más se presentan en la niñez y por tanto las patologías que se encuentran en las investigaciones que abordan el cáncer infantil ${ }^{(11)}$.

Se observa que entre el 80 y $85 \%$ de los cuidadores familiares de los grupos experimental y control son mujeres, hallazgo que robustece la evidencia frente a este aspecto, pues varios estudios han mostrado el papel protagónico de la mujer en la provisión de cuidados a personas en condición de cronicidad ${ }^{(12)}$.

El grado de escolaridad de los cuidadores familiares tiene niveles que oscilan entre la primaria y la secundaria, dato que es coherente con lo reportado por la literatura, pues la mayoría de cuidadores familiares con frecuencia tienen niveles medios a bajos de escolaridad ${ }^{(13-15)}$. El grado de escolaridad es un aspecto a considerar en la intervención de cuidadores familiares de niños con cáncer en su transición del rol, pues la preparación y conocimiento que tengan como condición de transición puede relacionarse con su grado de escolaridad. Además las intervenciones que se realicen sobre esta área, deben incluir información que los cuidadores familiares puedan comprender, que sea concreta y acorde a su escolaridad $^{(16,17)}$.
Las uniones maritales son el estado civil predominante en los cuidadores familiares, ya sea por unión marital de hecho o matrimonio. El estado civil del cuidador es un aspecto relevante en su transición del rol, por cuanto el contar con una pareja sentimental puede ser un factor relacionado con el apoyo percibido, así como con el afrontamiento y relevo, aspectos que influencian patrones de respuesta saludables a la transición ${ }^{(18)}$.

La ocupación de los cuidadores familiares es en mayor parte el hogar y trabajos independientes, hecho que revela la situación laboral de la mayoría de cuidadores de personas en situación de cronicidad. Los cuidadores familiares de niños con cáncer tienen un riesgo significativo de pérdida de empleo, de disminución de las oportunidades y horas de trabajo $^{(18)}$, situaciones que son evidentes en este estudio.

Este estudio pone a prueba una intervención de cuidado transicional de enfermería, que se condujo bajo la orientación conceptual de la teoría de rango medio de las transiciones propuesta por Afaf Meleis ${ }^{(2)}$, en donde a través de estrategias de enseñanza y aprendizaje del rol, que incluyeron instrucción intencional, ensayo e interacción con otros significativos, se buscó impactar en la competencia para el cuidado como patrón de respuesta a la transición del rol del cuidador del niño con cáncer. Esta metodología de intervención es consistente con otros estudios en los que aspectos del dominio del rol como el conocimiento ${ }^{(19-21)}$, el bienestar ${ }^{(22,23)}$ y la resolución de problemas ${ }^{(24-26)}$, son abordados con metodologías de interacción y entrenamiento del cuidador que aunque no explicitan enmarcarse en el rol, usan formas de intervención coherentes con la forma con que éste es aprendido.

Considerando que la competencia para cuidar no ha sido medida en otros estudios de intervención con cuidadores 
familiares de niños con cáncer, sumado a que estudios anteriores no reportan la magnitud del efecto, los resultados de este trabajo son comparables de forma parcial con otros adelantados. La intervención tuvo efecto de gran magnitud en el conocimiento, que incluye las nociones sobre la enfermedad, terapéuticas y actividades de cuidado físico, hallazgo similar al reportado en un estudio de intervención psicoeducacional $^{(21)}$. La interacción presencial con los cuidadores parece ser determinante para aumentar el conocimiento, pues en un estudio basado en web no reporta efecto sobre esta variable ${ }^{(20)}$.

Las condiciones personales que incluyen habilidades para resolver problemas, expresaron un efecto importante, similar al reportado en estudios en los que se brindó un entrenamiento específico en resolución de problemas ${ }^{(24-26)}$. El presente estudio abordó la resolución de problemas como una habilidad trasversal en el ejercicio del rol del cuidador, por lo que no se enfocó en hacer un entrenamiento específico para ello, sino lograr la habilidad a través de ejercicios de ensayo del rol individual y grupal.

La competencia instrumental de cuidado físico al niño también mejoró, resultado que se compara con el logrado en un estudio en el que sólo se brindó información sobre los cuidados físicos ${ }^{(27)}$. Futuros estudios podrían clarificar si para mejorar la competencia instrumental solo basta con brindar información o si mejores resultados pueden lograse con estrategias de ensayo del rol.

Una dimensión relevante en este estudio fue el bienestar, pues se parte de la premisa de que un cuidador no sólo debe ser competente para satisfacer las necesidades de su receptor de cuidados, sino que debe lograr un bienestar propio en el ejercicio de su rol. La intervención logró un efecto considerable sobre esta variable en el cuidador, comparable con el logrado con intervenciones de apoyo social en el entorno clínico brindada por enfermeras ${ }^{(22,23)}$.

El hallazgo central de esta investigación, es que la intervención de cuidado transicional de enfermería "Cuidando a nuestros niños con cáncer" tiene un efecto de gran magnitud en el aumento de la competencia para el cuidado en el cuidador familiar, lo que facilita su transición en el rol, en términos de la competencia para el cuidado. Este hallazgo tienen una importante implicación teórica y práctica, pues se pasa de un paradigma de intervención de enfermería basado en la transmisión de información a un cuidador para que ejecute con idoneidad acciones de cuidado directo al niño con cáncer, a considerar que las mismas se enmarcan en una construcción más amplia como es el ejercicio del rol de cuidador.

Al favorecer la competencia de cuidado en la transición del rol que vive el cuidador familiar del niño con cáncer, se logra un abordaje global, en el que no sólo son importantes los resultados en el niño, sino en el propio cuidador; considerando en este último aspectos como sus condiciones personales o disposición para cuidar, bienestar e interacciones sociales significativas.

\section{CONCLUSIONES}

Se comprueba de la intervención de cuidado transicional de enfermería "Cuidando a nuestros niños con cáncer" tiene un efecto de amplia magnitud sobre la competencia para el cuidado del cuidador familiar en el proceso de transición del rol.

La comprobación empírica de que la intervención de cuidado transicional de enfermería "Cuidando a nuestros niños con cáncer" aumenta la competencia para el cuidado en el cuidador familiar de niños con cáncer, la hace apta para ser aplicada en la práctica y replicada en la investigación. 


\section{REFERENCIAS BIBLIOGRÁFICAS}

1. Cline RJW, Harper FWK, Penner LA, Peterson AM, Taub JW, Albrecht TL. Parent communication and child pain and distress during painful pediatric cancer treatments. Soc Sci Med 2006;63:883-98. Doi: 10.1016/j.socscimed.2006.03.007

2. Meleis Al. Tansitions Theory - midle range and situation specific theories in nursing research and practice. New York. Springer Publishing Company; 2010. 664 p.

3. Gibbins J, Steinhardt K, Beinart H. A Systematic review of qualitative studies exploring the experience of parents whose child is diagnosed and treated for cancer. J Pediatr Oncol Nurs 2012;29:253-71. Doi: 10.1177/1043454212452791

4. Björk M, Wiebe T, Hallström I. Striving to survive: families' lived experiences when a child is diagnosed with cancer. J Pediatr Oncol Nurs 2015;22:265-75. Doi: 10.1177/1043454205279303

5. Fletcher PC. My child has cancer: the costs of mothers' experiences of having a child with pediatric cancer. Issues Compr Pediatr Nurs 2010;33:164-84. Doi: 10.3109/01460862.2010.498698

6. Meleis a I. Role insufficiency and role supplementation: a conceptual framework. Nurs Res 1975;24:264-71.

7. Meleis a I, Sawyer LM, Im EO, Hilfinger Messias DK, Schumacher K. Experiencing transitions: an emerging middle-range theory. ANS Adv Nurs Sci 2000;23(1):1228.

8. James K, Keegan-Wells D, Hinds PS, Kelly KP, Bond D, Hall B, et al. The Care of My Child with Cancer: Parents' Perceptions of Caregiving Demands. J Pediatr Oncol Nurs. 1 de noviembre de 2002;19:218-28. Doi: 10.1053/jpon.2002.128777

9. Aburn G, Gott M. Education given to parents of children newly diagnosed with acute lymphoblastic leukemia: A narrative review. J Pediatr Oncol Nurs 28:300-5. Doi: $10.1177 / 1043454211409585$
10. Carrillo GM, Herrera BS, Rojas EMA. Validation of an instrument to assess the homecare competency of the family caregiver of a person with chronic disease [Internet]. Investigación y Educación en Enfermería. 2015 [Acceso 26 de enero de 2016]. Disponible en: http://aprendeenlinea.udea.edu.co/revistas/index.php/iee/ article/view/24453/20449

11. Stehl ML, Kazak $A E$, Alderfer $M$ a, Rodriguez A, Hwang W, Pai ALH, et al. Conducting a randomized clinical trial of an psychological intervention for parents / caregivers of children with cancer shortly after diagnosis. J Pediatr Psychol. 2009;34:80316. Doi: 10.1093/jpepsy/jsn130

12. Ballestas Cueto H, López Ortega E, Meza Bustillo R, Palencia Suarez K, Ramos Polo D, Montalvo Prieto AA. Cuidadores familiares de niños con cáncer y su funcionalidad. Rev Científica Salud Uninorte [Internet]. 2013 [Acceso 8 de julio de 2015];29(2). Disponible en: http://rcientificas.uninorte.edu.co/index.php/salud/article/view/3969

13. Barrera L, Galvis C, Moreno ME, Pinto N, Rocha L, González R, et al. La habilidad de cuidado de los cuidadores familiares de personas con enfermedad crónica. Investig Educ Enferm 2006;24:36-46.

14. Montalvo Prieto AA, Badrán Navarro Y, Cavadía Puello C, Medina Ripoll E, Méndez Soraca K, Padilla Lobo C, et al. Habilidad de cuidado de cuidadores familiares principales de pacientes con ACV. Cartagena (Colombia). Rev Científica Salud Uninorte. 2010;26(2).

15. Carrillo González GM, Ortiz LB, Herrera BS, Carreño SP, Díaz LC. Efecto del programa de habilidad de cuidado para cuidadores familiares de niños con cáncer. Rev Colomb Cancerol [Internet]. 2013;17:168.

16. Maree JE, Parker S, Kaplan L, Oosthuizen J. The information needs of south african parents of children with cancer. J Pediatr Oncol Nurs 2015;33:9-17. Doi: 10.1177/1043454214563757 
17. Njuguna F, Mostert S, Seijffert A, Musimbi J, Langat $S$, van der Burgt RHM, et al. Parental experiences of childhood cancer treatment in Kenya. Support Care Cancer 2015;23:1251-9. Doi: 10.1007/s00520014-2475-x

18. Lau S, Lu X, Balsamo L, Devidas M, Winick N, Hunger SP, et al. Family life events in the first year of acute lymphoblastic leukemia therapy: a children's oncology group report. Pediatr Blood Cancer 2014;61:2277-84. Doi: 10.1002/ pbc. 25195

19. Ringnér A, Karlsson S, Hällgren Graneheim $U$. A person-centred intervention for providing information to parents of children with cancer. Experiences and effects. Eur J Oncol Nurs 2015;19:318-24. Doi: 10.1016/j.ejon.2014.10.012

20. Dragone MA, Bush PJ, Jones JK, Bearison DJ, Kamani S. Development and evaluation of an interactive CD-ROM for children with leukemia and their families. Patient Educ Couns 2002;46:297-307.

21. Othman A, Blunden S, Mohamad N, Hussin ZAM, Osman ZJ. Piloting a psychoeducation program for parents of pediatric cancer patients in Malaysia. Psychooncology. 2010;19:326-31. Doi: 10.1002/ pon. 1584

22. Svavarsdottir EK, Sigurdardottir A. The feasibility of offering a family level intervention to parents of children with cancer.
Scand J Caring Sci 2005;19:368-72. Doi: 10.1111/j.1471-6712.2005.00360.x

23. Svavarsdottir EK, Sigurdardottir AO. Developing a family-level intervention for families of children with cancer. Oncol Nurs Forum 2006;33:983-90. Doi: 10.1188/06. ONF.983-990

24. Sahler OJZ, Varni JW, Fairclough DL, Butler RW, Noll RB, Dolgin MJ, et al. Problemsolving skills training for mothers of children with newly diagnosed cancer: a randomized trial. J Dev Behav Pediatr 2002;23:77-86.

25. Sahler OJZ, Fairclough DL, Phipps S, Mulhern RK, Dolgin MJ, Noll RB, et al. Using problem-solving skills training to reduce negative affectivity in mothers of children with newly diagnosed cancer: report of a multisite randomized trial. J Consult Clin Psychol2005;73:272-83. Doi: 10.1037/0022-006X.73.2.272

26. Sahler OJZ, Dolgin MJ, Phipps S, Fairclough DL, Askins MA, Katz ER, et al. Specificity of problem-solving skills training in mothers of children newly diagnosed with cancer: Results of a multisite randomized clinical trial. J Clin Oncol 2013;31:132935. Doi: 10.1200/JCO.2011.39.1870

27. Caliskan M, Ozsoy S. Effectiveness of a discharge-planning program and home visits for meeting the physical care needs of children with cancer. Support Care Cancer 2010;18:243-53. Doi: 10.1007/s00520009-0650-2 